\title{
EXPLOSIVE SOLUTIONS OF A STOCHASTIC NON-LOCAL REACTION-DIFFUSION EQUATION ARISING IN SHEAR BAND FORMATION
}

\author{
NIKOS I. KAVALLARIS \\ Dedicated to Professor Messoud Efendiev on the occasion of his 60th birthday
}

\begin{abstract}
In this paper, we consider a non-local stochastic parabolic equation which actually serves as a mathematical model describing the adiabatic shear-banding formation phenomena in strained metals. We first present the derivation of the mathematical model. Then we investigate under which circumstances a finite-time explosion for this non-local SPDE, corresponding to shear-banding formation, occurs. For that purpose some results related to the maximum principle for this non-local SPDE are derived and afterwards the Kaplan's eigenfunction method is employed.
\end{abstract}

\section{INTRODUCTION}

In the current work we consider the following non-local stochastic parabolic problem

$$
\begin{aligned}
& \frac{\partial u}{\partial t}=\Delta u+F(u)+\sigma(u) d W,(x, t) \in D_{T}:=D \times(0, T), \\
& u(x, t)=0, \quad x \in \partial D \times(0, T), \\
& u(x, 0)=\xi(x), \quad x \in D,
\end{aligned}
$$

where $T>0$ and $D$ is a bounded subset of $\mathbb{R}^{d}, d=1,2$, with smooth boundary and $\xi: D \rightarrow \mathbb{R}^{+}$is almost surely in class $L^{2}(D)$ and not identical zero. Besides the non-local term $F(u)$ is defined by

$$
F(u):=\frac{\lambda e^{u}}{\left(\int_{D} e^{u} d x\right)^{p}},
$$

for some positive constants $\lambda$ and $p$, whereas $\sigma(u)$ is a (local) function of $u$ whose growth will be identified later. Here $d W$ denotes by convention the formal (time) derivative of the Wiener random process $W(t)$ which it will be determined more rigorously below.

Our motivation for studying problem (1.1)-(1.3) is that it can be used as a mathematical model to describe a phenomenon called shear-banding formation which occurs in high strained metals. The derivation of this mathematical model is illustrated in Section 2 whereupon the presence of the multiplication noise term $\sigma(u) d W$ is also fully justified.

Date: May 29, 2015.

1991 Mathematics Subject Classification. Primary 60H15, 35B44 ; Secondary 34B10, 35B50, 35B51.

Key words and phrases. Non-local, Stochastic Partial Differential Equations, Maximum principle, Blow-up, Shear band formation. 
In the following we introduce the functional setting in order to interpret problem (1.1)(1.3) as an abstract Itô equation in a proper Hilbert space. The Itô-formulation is actually more appropriate for the presentation of most of our results.

Let $(\Omega, \mathscr{F}, \mathbb{P})$ be a probability space and let $\left\{\mathscr{F}_{t}: t \in[0, T]\right\}$ be a family of sub$\sigma$-algebras of $\mathscr{F}$ building a filtration.

Let also $H=L^{2}(D)$ be the Hilbert space equipped with an inner product and norm denoted by $\langle\cdot, \cdot\rangle$ and $\|\cdot\|$ respectively.

In the sequel, we recall the definition of Hölder spaces with different regularity in time and space denoted by $C^{\alpha, \beta}(\bar{D} \times[0, T])$, for $0<\alpha, \beta<1$. Indeed, this space is equipped with the norm

$$
\|f\|_{C^{\alpha, \beta}}=\sup _{(x, t)}|f(x, t)|+\sup _{(x, t) \neq(y, s)} \frac{|f(x, t)-f(y, s)|}{|x-y|^{\alpha}+|t-s|^{\beta}} .
$$

Following the standard modification we can also employ the Hölder spaces $C^{\alpha, \beta}(\bar{D} \times[0, T])$, for $\alpha, \beta \geq 1$.

Moreover for each $t>0$ and for all real numbers $p, q \geq 1$ we define the space

$$
L^{p, q}(D \times[0, t])=\left\{h:\|h\|_{p, q, t}:=\left(\int_{0}^{t}\|h(\cdot, s)\|_{p}^{q} d s\right)^{1 / q}<\infty\right\} .
$$

In the limiting case $p=q=\infty$ we define

$$
L^{\infty, \infty}(D \times[0, t])=\left\{h:\|h\|_{\infty, \infty, t}:=\operatorname{ess~sup~}_{s \in[0, t]}\|h(\cdot, s)\|_{\infty}<\infty\right\} .
$$

For some parameter $\theta \in[0,1)$ and for space dimension $d=1,2$ we define

$$
\Gamma_{\theta}^{*}=\left\{(p, q) \in[1, \infty]^{2}: \frac{2^{*}}{2^{*}-2} \frac{1}{p}+\frac{1}{q}=1-\theta\right\}
$$

where $2^{*}=\frac{2 d}{d-2}$. While for $d \geq 3$ we define

$$
\Gamma_{\theta}^{*}=\left\{(p, q) \in[1, \infty]^{2}: \frac{d}{2 p}+\frac{1}{q}=1-\theta\right\} .
$$

In each case we define the functional space

$$
L_{\theta}^{*}=\sum_{(p, q) \in \Gamma_{\theta}^{*}} L^{p, q}(D \times[0, t])
$$

endowed with the norm

$$
\|h\|_{\theta ; t}^{*}:=\inf \left\{\sum_{i=1}^{n}\left\|h_{i}\right\|_{p_{i}, q_{i} ; t}: h=\sum_{i=1}^{n} h_{i}, h_{i} \in L^{p_{i}, q_{i}}(D \times[0, t]),\left(p_{i}, q_{i}\right) \in \Gamma_{\theta}^{*}, i=1, \ldots, n ; n \geq 1\right\} .
$$

For more details on this function space see Appendix in [10]. 
We consider $W(t)$ to be a continuous $N$-dimensional Wiener process in the complete probability space $(\Omega, \mathscr{F}, \mathbb{P})$. More precisely for any positive integer $N$ we define

$$
W(t)=\sum_{k=1}^{N} e_{k} \beta_{k}(t)
$$

where $\beta_{k}(t), k=1,2,3, \ldots N$ are independent standard $\mathbb{R}$-valued Brownian motions in one dimension and $\left(e_{k}\right)_{k=1}^{N}$ is an orthonormal basis of $\mathbb{R}^{N}$.

If we now define the linear operator $A=\Delta: \mathscr{D}(A)=H \rightarrow H$ then the solution $u(x, t ; \omega)$ (for any $\omega \in \Omega$ ) of problem (1.1)-(1.3) can be considered as the predictable stochastic process in the Hilbert space $H$ satisfying the following Itô-type stochastic differential equation

$$
\begin{aligned}
& d u(\cdot, t ; \omega)=(A u(\cdot, t ; \omega)+F(u(\cdot, t ; \omega)) d t+\sigma(u(\cdot, t ; \omega)) d W(t ; \omega), \quad t>0, \\
& u(\cdot, 0 ; \omega)=\xi(\omega) .
\end{aligned}
$$

Henceforth, for simplicity we omit $\omega$ without any confusion. Therefore we can formulate (1.5)-(1.6) equivalently in $H$ as a stochastic integral equation

$$
u(\cdot, t)=\xi+\int_{0}^{t}[A u(\cdot, s)+F(u(\cdot, s))] d s+\int_{0}^{t} \sigma(u(\cdot, s)) d W(s),
$$

where the last integral in (1.7) is a well defined stochastic integral, see also [6].

A strong solution $u$ to (1.1)-(1.3) (or equivalently for (1.5)-(1.6) and for (1.7)) belongs to $C([0, T] ; H) \cap L^{2}\left((0, T) ; H^{1}(D)\right)$ for some $T>0$ almost surely (a.s.), i.e.

$$
P\left(u \in C([0, T] ; H) \cap L^{2}\left((0, T) ; H^{1}(D)\right)\right)=1 .
$$

Under the assumption that $\xi(x) \geq 0$ a.s. in $D$ we can deduce that $u$ is almost surely (a.s.) positive in $D_{T}$ by a comparison theorem, see $[1,11,12,22]$. Therefore henceforth we focus on the study of positive solutions of problem (1.1)-(1.3).

To avoid finite-time explosion due to the stochastic term as well as some extra technical difficulties we assume that $\sigma: H \rightarrow H$ is a Lipschitz continuous with linear growth. A finite-time explosion due to the stochastic term will be investigated in a forthcoming paper. Moreover the Lipschitz continuity of the function $f(s)=e^{s}$ implies the Lipschitz continuity of the operator operator $\tilde{F}: H \rightarrow H$ defined by

$$
\tilde{F}(u)(x):=F(u(x))=\frac{\lambda e^{u(x)}}{\left(\int_{D} e^{u(x)} d x\right)^{p}},
$$

and overall the existence of a local-in-time strong solution is guaranteed which is actually even more regular. Indeed, it is proven in [18], following the approach developed in [9], that for any $k \in \mathbb{N}$ and $\mu \in(0,1 / 2)$ there exists $\gamma>0$ such that

$$
u \in L^{m}\left(\Omega ; C^{\mu, k+\gamma}\left(\overline{D_{T}}\right)\right), \quad m \geq 2,
$$

provided the initial data are regular enough. 
The main purpose of the current work is to investigate under which circumstances a global-in-time solution of the non-local stochastic parabolic problem (1.5)-(1.6) ceases to exist. In such a case we say that the solution $u$ to (1.5)-(1.6) explodes (or blows up) in finite time.

According to the definition in $[15$, p. 172] the solution to the problem (1.5)-(1.6) explodes in finite time if $\mathbb{P}\left[T_{e}<\infty\right]=1$ where $T_{e}$ is the explosion time defined as $T_{e}=\inf \left\{t>0:\|u(\cdot, t)\|_{\infty}=\infty\right\}$. However in the current work we provide an alternative definition which is more convenient for our approach.

Definition 1.1. The solution u of problem (1.5)-(1.6) explodes in finite time if there exists $0 \leq T_{e}<\infty$ such that

$$
\limsup _{t \rightarrow T_{e}} \mathbb{E}\left[\|u(\cdot, t)\|_{\infty}\right]=\infty,
$$

where $\mathbb{E}$ stands for the expectation of the $H$-valued stochastic process $u$.

Apart from its own mathematical interest, since it determines the occurrence of a singularity in time, explosion for solutions (1.5)-(1.6) is also very important from the applications point of view as we will see in the next section.

To our knowledge there are no available explosive results for the non-local stochastic problem (1.5)-(1.6) in the literature, although there some works investigating the finitetime blow up for local stochastic reaction-diffusion equations, [7, 8, 13].

The deterministic case, whereupon $u$ satisfies the non-local parabolic problem

$$
\begin{aligned}
& \frac{\partial u}{\partial t}=\Delta u+\frac{\lambda f(u)}{\left(\int_{D} f(u) d x\right)^{p}},(x, t) \in D_{T}, \\
& u(x, t)=0, \quad x \in \partial D \times(0, T), \\
& u(x, 0)=\xi(x), \quad x \in \Omega,
\end{aligned}
$$

for $0<p<1$ and $D$ is a convex domain of $\mathbb{R}^{d}, d \geq 1$, was investigated in [17]. In particular, the authors proved that the solution $u(x, t)$ to (1.9)-(1.11) blows up in finite time either for big values of the parameter $\lambda$ or for large enough initial data (Theorems 1 and 2 in [17]), under the assumptions that the function $f(s)$ is positive, increasing and convex satisfying

$$
\int_{b}^{\infty} \frac{d s}{f^{1-p}(s)} d s<\infty \text { for } \quad b>0 .
$$

The arguments employed in [17] are adapted here to the treat the case of the nonlocal stochastic parabolic problem (1.5)-(1.6), though some technical results, are not available in the literature of SPDEs, should be proved first.

The structure of the paper is as follows. Section 2 is devoted to the derivation of the mathematical model (1.1)-(1.3) which describes the phenomenon of shear band formation in the high strain-rate metals. In section 3 some auxiliary results like maximum principle and Hopf's lemma for SPDEs are provided. The main results of the current paper, 
associated with the finite-time blow-up of (1.1)-(1.3), are presented and proven in section 4 .

\section{Derivation of the Model}

The main purpose of this section is to associate the mathematical problem (1.1)-(1.3) to the phenomenon of shear band formation in the high strain-rate torsion test, see $[3,4]$. The mathematical model (1.1)-(1.3) actually describes the localization of plastic strain into adiabatic shear bands during rapid plastic shearing of metals. Shear band formation is caused by the heat generated in regions with the highest strain rate. With insufficient time for diffusion of the produced heat, a localized thermal softening of the metal occurs which enhances plastic flow in a thin zone. In the following, we proceed to the derivation of the mathematical model in dimensions $d=1,2$ describing the above process by adopting the approach in [2].

Assume loading a thin-walled tube of metal of length $d$ in torsion with ends kept at constant temperature $\Theta_{0}$ and with tube having initial temperature $\Theta_{0}$. The one end of the tube held motionless while the other end is twisted at a constant rate $v=v_{0}$. Let $z$ represent the axial coordinate, $t$ the time, $w(z, t)$ the linear displacement, $v(z, t)=w_{t}(z, t)$ the velocity, $\gamma(z, t)=w_{z}(z, t)$ the shear strain, and $\tau(z, t)$ the shear stress, then the thermovisco-plastic shear model is described by the following system of conservation laws:

$$
\left.\begin{array}{rl}
\rho v_{t} & =\tau_{z}, \\
\delta \tau_{t} & =v_{z}-\gamma_{t}, \\
\Theta_{t} & =\xi \Theta_{z z}+\tau \mu^{p-1} \gamma_{t}, \\
\gamma_{t} & =\Phi(\tau, \gamma, \Theta),
\end{array}\right\}
$$

where $\rho, \delta, \chi, p$ stand for some constants and $\mu=\mu(t)$ is a parameter controlling the whole process.

In particular, the first equation of (2.1) describes the conservation of momentum, while the second one is a constitutive equation which governs the evolution of the shear stress $\tau$. Besides, the third equation describing the evolution of temperature $T$ is actually the balance of energy equation of the system. Here, it is assumed that all of the plastic work is dissipated. Finally, the last equation of (2.1) is an internal-variable type of constitutive equation for the evolution of the effective plastic shear strain $\gamma$.

If $\rho \ll 1$ and $\delta \ll 1$, which is actually a realistic assumption as it was pointed out by Burns in [3] regarding the experiments of Marchand and Duffy ([20]), then (2.1) is reduced to the quasi-static model

$$
\left.\begin{array}{rl}
\tau_{z} & =0, \\
v_{z} & =\gamma_{t}, \\
\Theta_{t} & =\xi \Theta_{z z}+\tau \mu^{p-1} \gamma_{t}, \\
\gamma_{t} & =\Phi(\tau, \gamma, \Theta),
\end{array}\right\}
$$


from which we recognize that the stress is only time-dependent, i.e. $\tau=\tau(t)$. Assuming that the stress-stain is in the plastic regime $([20])$, then $\tau=\tau(t)=\tau_{0}$ is approximately constant as long as the temperature $\Theta$ does not increase rapidly. In the case that the temperature is affected by environmental fluctuations then we can perturb the third equation of (2.2) by adding term of the form $\alpha \eta(x, t)$ where $\alpha$ is a coefficient measuring the intensity of the noise term $\eta(x, t)$. The addition of this noise term can be also conceived as a lack of knowledge of certain physical parameters of the system. It is quite realistic to consider that the coefficient $\alpha$ should depend on the temperature $\Theta$, whereas the noise $\eta(x, t)$ could be taken to be white noise i.e. $\eta(x, t)=C \dot{b}(t)$ for a given positive constant $C$ where by convention $\dot{b}(t)$ stands for the formal time derivative of the Brownian motion $b(t)$, see also $[2,5]$. Henceforth in this section the stochastic noise term $\alpha(\Theta) C \dot{b}(t)$ will be denoted for simplicity by $S N T$.

We can consider that the (plastic) strain rate $\gamma$ is given by the Arrhenius law

$$
\gamma_{t}=v_{z}=\mu \exp \left(\frac{-\Delta G\left(\tau_{0}\right)}{K \Theta}\right)
$$

where, under proper scaling, the parameter $\mu$ is the one appearing into (2.2) while $\Delta G$ is the activation enthalpy for the plastic deformation process and $K$ is the constant of Boltzman's law. Equation (2.3) is actually inspired by consideration of microstructural physical processes.

Then system (2.2) simplifies to the system

$$
\begin{aligned}
\Theta_{t} & =\xi \Theta_{z z}+\tau_{0} \mu^{p} \exp \left(\frac{-\Delta G\left(\tau_{0}\right)}{K \Theta}\right)+S N T, \\
v_{z} & =\mu \exp \left(\frac{-\Delta G\left(\tau_{0}\right)}{K \Theta}\right), \\
\Theta(0, t) & =\Theta(d, t)=\Theta_{0}, \quad v(0, t)=0, \quad v(d, t)=v_{0}, \\
\Theta(z, 0) & =\Theta_{0} .
\end{aligned}
$$

Integrating the second equation of $(2.4)$ over $(0, d)$ and using the boundary conditions satisfied by $v$ we obtain

$$
\mu=\mu(t)=\frac{v_{0}}{\left(\int_{0}^{d} e^{\frac{-\Delta G\left(\tau_{0}\right)}{K \Theta}} d z\right)} .
$$

Therefore the system (2.4) is reduced to the following initial value boundary value problem for a non-local stochastic heat equation

$$
\left.\begin{array}{rl}
\Theta_{t} & =\xi \Theta_{z z}+\tau_{0} v_{0}^{p} \frac{e^{\frac{-\Delta G\left(\tau_{0}\right)}{K \Theta}}}{\left(\int_{0}^{d} e^{\frac{-\Delta G\left(\tau_{0}\right)}{K \Theta}} d z\right)^{p}}+S N T \\
\Theta(0, t) & =\Theta(d, t)=\Theta_{0} \\
\Theta(z, 0) & =\Theta_{0} .
\end{array}\right\}
$$


Now rescaling the spatial and time variables (2.6) reads

$$
\left.\begin{array}{rl}
\Theta_{t} & =\Theta_{z z}+\frac{\hat{\lambda} e^{\frac{-\Delta G\left(\tau_{0}\right)}{K \Theta}}}{\left(\int_{-1}^{1} e^{\frac{-\Delta G\left(\tau_{0}\right)}{K \Theta}} d z\right)^{p}}+S N T \\
\Theta(-1, t) & =\Theta(1, t)=\Theta_{0} \\
\Theta(z, 0) & =\Theta_{0}
\end{array}\right\},
$$

where

$$
\hat{\lambda}=\frac{2^{p-2} v_{0}^{p} \tau_{0}}{\omega d^{p-2}}
$$

Employing the dimensionless variables

$$
u=\frac{\Delta G\left(\tau_{0}\right)}{K \Theta_{0}^{2}}\left(\Theta-\Theta_{0}\right), \quad \varepsilon=\frac{K \Theta_{0}}{\Delta G\left(\tau_{0}\right)},
$$

and using the identity

$$
\frac{u}{1+\varepsilon u}=\frac{\Delta G\left(\tau_{0}\right)}{K}\left(\frac{1}{\Theta_{0}}-\frac{1}{\Theta}\right)=\frac{1}{\varepsilon}\left(\frac{1}{\Theta_{0}}-\frac{1}{\Theta}\right)
$$

we derive the dimensionless problem

$$
\left.\begin{array}{rl}
u_{t} & =u_{z z}+\frac{\lambda e^{u /(1+\varepsilon u)}}{\left(\int_{-1}^{1} e^{u /(1+\varepsilon u)} d z\right)^{p}}+S N T \\
u(-1, t) & =u(1, t)=0 \\
u(z, 0) & =0
\end{array}\right\}
$$

where

$$
\lambda=\frac{\hat{\lambda} e^{-(1-p) / \varepsilon}}{\varepsilon \Theta_{0}} .
$$

From the experimental study of shear banding formation, see [20], it is known that $T$ ranges from $3000 K$ to $8000 K$ so that the nondimensional temperature $u$ takes values in the interval $[0,70]$, when $\varepsilon$ is approximately equal to 0.025 and therefore model (2.7) is reduced to

$$
\left.\begin{array}{rl}
u_{t} & =u_{z z}+\frac{\lambda e^{u}}{\left(\int_{-1}^{1} e^{u} d z\right)^{p}}+S N T \\
u(-1, t) & =u(1, t)=0 \\
u(z, 0) & =0
\end{array}\right\} .
$$

A two-dimensional mathematical problem can model the configuration, when the tube is long and paralell to the $z$-direction, whereas its cross section is unchangeable, e.g. the tube is cylindrical or prismatic (or approximately so). The cross section of the cylinder is $Q \subset \mathbb{R}^{2},((x, y) \in Q$ if $(x, y, z) \in \partial D)$ with the diameter of $Q$ much less than $L$, the 
length of the tube. Now the thermovisco-plastic shear model across the cross section $Q$ is described by the following system of conservation laws:

$$
\begin{aligned}
\rho v_{t} & =\tau_{x y}, \\
\delta \tau_{t} & =v_{x y}-\gamma_{t}, \\
\Theta_{t} & =\xi\left(\Theta_{x x}+\Theta_{y y}\right)+\tau \mu^{p-1} \gamma_{t}+S N T \\
\gamma_{t} & =\Phi(\tau, \gamma, \Theta) .
\end{aligned}
$$

Assuming again that $\rho, \delta \ll 1$ as well as that temperature is zero on the curved surface of $D$ and following the same steps as above we derive the two-dimensional model

$$
\begin{aligned}
u_{t} & =\Delta u+\frac{\lambda e^{u}}{\left(\int_{Q} e^{u} d x\right)^{p}}+S N T, \quad x \in Q, t>0, \\
u(x, t) & =0, x \in \partial Q, t>0, \\
u(x, 0) & =0, x \in Q,
\end{aligned}
$$

where $x$ stands for the position in the cross-section $Q$ and $\Delta$ denote the two dimensional Laplacian, ignoring also any side effects.

During the experimental study of the shear band formation, [20], it was observed that under very high temperatures a narrow shear band is formed. This narrow band of high strain often precedes the failure in materials. Therefore, engineers are very interested in constructing mathematical models could predict the occurrence of such shear bands.

From the above analysis it is clear that the occurrence of shear bands is strictly connected with the thermal runaway of the temperature $T$ of the twisted thin-walled tube. Thus, the main question is: Does the non-local stochastic model (2.7) predict thermal runaway, and hence shear-band formation, confirming the experimental observations?

The answer to this question is given in Section 4, where the occurrence of explosive solutions of (1.1)-(1.2) is proven.

\section{Auxiliary Results}

The proof of the blow-up results presented in section 4 is essentially based on the control of the solution near the boundary. This is obtained by employing the moving plane method which actually needs the validity of a Hopf maximum principle result for parabolic SPDEs. Thereupon in this section, we provide such a result, which apart from its auxiliary role in the proofs of Theorems 4.1 and 4.2 , it has also its own importance in the context of the study of parabolic SPDEs. As far as we know, it is the first time that a Hopf maximum is proved in the literature.

For reader's convenience we first recall some required definition and the Hopf maximum principle for the deterministic case, see also [14, 21]. 
Definition 3.1. Let $P_{0}=\left(x_{0}, t_{0}\right)$ be a point on the boundary of $D_{T}$. If there exists a closed ball $B$ centered at $(\bar{x}, \bar{t})$ such that

$$
B \subset \bar{D}_{T}, \quad B \cap \partial D_{T}=\left\{P_{0}\right\}, \bar{x} \neq x_{0},
$$

then we say that $P_{0}$ has the inside strong sphere property.

Theorem 3.2. Let $\mathscr{H}$ denote the heat operator $\mathscr{H}(u):=\Delta u-\frac{\partial u}{\partial t}$. Assume that $\mathscr{H}(u) \geq 0$ and $\max u=M$ is attained at $P_{0} \in \partial D_{T}$, i.e. $u\left(P_{0}\right)=M$, where $P_{0}$ has the inside strong sphere property. Further assume that for some neighbourhood $\mathscr{V}$ of $P_{0}$,

$$
u<M, \text { in } D_{T} \cap \mathscr{V} .
$$

Then we have

$$
\frac{\partial u}{\partial \nu}\left(P_{0}\right)<0
$$

where $\nu\left(P_{0}\right)=\nu$ is the outer normal direction at $P_{0}$.

Now we provide a proof of a version of the maximum principle associated with semilinear SPDEs following the approach in [10].

Theorem 3.3. (Maximum Principle) Let $V(x, t ; \hat{\xi})$ be the solution of the following SPDE

$$
\begin{aligned}
& d V(\cdot, t)=[A V(\cdot, t)+G(V(\cdot, t))] d t+\chi(V(\cdot, t)) d W(t), \quad 0<t<T, \\
& V(\cdot, 0)=\hat{\xi} \geq 0 \text { a.s., }
\end{aligned}
$$

in $D_{T}=D \times(0, T)$ for any bounded domain $D$ in $\mathbb{R}^{d}, d \geq 1$ and any $T>0$. Assume further that $G: H \rightarrow H$ and $\chi: H \rightarrow H$ are Lipschitz continuous functions. If $M=$ $M_{T}:=\max _{x \in \partial D_{T}} V(x, t)$ then

$$
V(x, t) \leq M, \quad \text { for any } \quad(x, t) \in \bar{D}_{T} .
$$

Proof. Set $M_{t}=\max _{x \in \partial D_{t}} V(x, t)$ where $D_{t}:=D \times(0, t)$. Note that $z(x, t)=V(x, t)-M_{t}$ satisfies

$$
\begin{aligned}
& d z(\cdot, t)=[A z(\cdot, t)+\widehat{G}(z(\cdot, t))] d t+\widehat{\chi}(z(\cdot, t)) d W(t), \quad 0<t<T, \\
& z(\cdot, 0)=\hat{\xi}-\|\hat{\xi}\|_{\infty},
\end{aligned}
$$

where $\widehat{G}(z):=G\left(z+M_{t}\right)-G\left(M_{t}\right)$ and $\widehat{\chi}(z):=\chi\left(z+M_{t}\right)-\chi\left(M_{t}\right)$, since $M_{t}$ solves the following $\mathrm{SDE}$

$$
\begin{aligned}
& d M_{t}=G\left(M_{t}\right) d t+\chi\left(M_{t}\right) d W(t), \quad 0<t<T, \\
& M_{0}=\|\hat{\xi}\|_{\infty} .
\end{aligned}
$$

Then by virtue of Theorem 18 in [10] we get

$$
\mathbb{E}\left[\left\|z^{+}\right\|_{\infty, \infty ; t}^{2}\right] \leq s(t) \mathbb{E}\left[\left\|\left(\hat{\xi}-M_{0}\right)^{+}\right\|_{\infty}^{2}+\left(\|\widehat{G}(0)\|_{\theta ; t}^{*}\right)^{2}+\left\|\widehat{\chi}^{2}(0)\right\|_{\theta ; t}^{*}\right],
$$


for some $s(t)>0$. Since $\widehat{G}(0)=\widehat{\chi}(0) \equiv 0$, we finally derive that

$$
\mathbb{E}\left[\left\|z^{+}\right\|_{\infty, \infty ; t}^{2}\right]=0
$$

where $z^{+}$stands for the positive part of $z$.

The latter implies that $z(x, t) \leq M_{t} \leq M_{T}$ for any $(x, t) \in \bar{D}_{T}$ and this completes the proof of the Theorem.

Now we are ready to proceed towards the proof of Hopf maximum principle.

Theorem 3.4. (Hopf's Maximum Principle) Let $u$ be a solution of problem (1.1)-(1.3). Assume also that $\max _{\bar{D}_{T}} u=M_{T}=M$ is attained at $P_{0}=\left(x_{0}, t_{0}\right) \in \partial D_{T}$, where $P_{0}$ has the inside strong sphere property, and there is some neighbourhood $\mathscr{V}$ of $P_{0}$, such that

$$
u<M, \quad \text { in } D_{T} \cap \mathscr{V} .
$$

Then

$$
\frac{\partial u}{\partial \nu}\left(P_{0}\right)<0
$$

Proof. By Definition 3.1 there exists a closed ball $B$ centered at $(\bar{x}, \bar{t})$ such that

$$
B \subset \bar{D}_{T}, \quad B \cap \partial D_{T}=\left\{P_{0}\right\}, \bar{x} \neq x_{0} .
$$

We assume the interior of $B$ lies in $D_{T} \cap \mathscr{V}$ and denote the boundary of $B$ by $S$. Let $\pi$ be a hyperplane which divides the $(x, t)$-plane into two half-planes $\pi^{-}$and $\pi^{+}$such that $(\bar{x}, \bar{t}) \in \pi^{-}$and $\left(x_{0}, t_{0}\right) \in \pi^{+}$.

Since $\bar{x} \neq x_{0}$, we can choose $\pi$ such that $B^{+}=\pi^{+} \cap B$ is not empty and such that $|\bar{x}-x|>$ const $>0$, for any $(x, t) \in B^{+}$. The boundary of $B^{+}$consists of one part $C_{1}$ lying on $S$ and another part $C_{2}$ lying on $\pi$.

Introduce the function

$$
h(x, t)=e^{-\beta\left(|x-\bar{x}|^{2}+(t-\bar{t})^{2}\right)}-e^{-\beta R^{2}},
$$

where $R$ is the radius of $S$. We then have

$$
\begin{aligned}
& h=0 \text { on } C_{1}, \\
& h \geq 0 \text { on } B^{+}, \\
& \mathscr{H}(h)>0 \quad \text { in } B^{+} \text {for } \beta \text { sufficiently large. }
\end{aligned}
$$

Set

$$
V=u+\varepsilon h,
$$

then it is easy to see that

$$
\begin{aligned}
& V<M \quad \text { on } C_{2}, \\
& V(P)=u(P)<M \quad \text { on } C_{1}, \text { if } P \neq P_{0}, \\
& V\left(P_{0}\right)=u\left(P_{0}\right)=M .
\end{aligned}
$$


Moreover, since $h$ solves the heat equation, i.e. $d h(\cdot, t)=\Delta h(\cdot, t) d t$, then $V$ satisfies

$$
\begin{aligned}
d V(\cdot, t) & =[A V(\cdot, t)+F(V(\cdot, t)-\varepsilon h(\cdot, t))] d t+\sigma(V(\cdot, t)-\varepsilon h(\cdot, t)) d W(t) \\
& =[A V(\cdot, t)+\widetilde{F}(V(\cdot, t))] d t+\widetilde{\sigma}(V(\cdot, t))) d W(t)
\end{aligned}
$$

where

$$
\widetilde{F}(V)=F(V-\varepsilon h), \quad \widetilde{\sigma}(V)=\sigma(V-\varepsilon h) .
$$

By the assumptions on functions $F$ and $\sigma$ and by virtue of Theorem 3.3, we deduce

$$
V(x, t) \leq \max _{\partial D_{T}} V=M, \quad \text { for any } \quad(x, t) \in B^{+} .
$$

Thus, since due to (1.8) $V$ is regular enough, we have

$$
\frac{\partial V}{\partial \nu}\left(P_{0}\right) \leq 0
$$

or equivalently

$$
\frac{\partial u}{\partial \nu}+\varepsilon \frac{\partial h}{\partial \nu} \leq 0, \quad \text { at } P_{0}
$$

However, by virtue of Theorem 3.2 we have $\frac{\partial h}{\partial \nu}<0$ at $P_{0}$, thereupon we finally obtain

$$
\frac{\partial u}{\partial \nu}\left(P_{0}\right)<0
$$

Similarly to the deterministic case, see [17], in order to tackle the technical difficulties arising due to the presence of the non-local term $K(t)=\left(\int_{D} e^{u(x, t)} d x\right)^{-p}$, we need to estimate the contribution of $u(x, t)$ near the boundary of $D$. For that purpose we use the moving plane method similarly to $[17,19]$. Although most of the applied arguments are quite standard in the context of deterministic PDEs, since it is the first time, according to our knowledge, that those ideas used for SPDEs, a detailed proof is provided. More precisely, the following holds.

Lemma 3.5. Let $u$ be the solution of (1.5)-(1.6) and $\xi \in L^{2}\left(\Omega ; L^{2}(D)\right), \xi \geq 0$ a.s. in D. If $D \subset \mathbb{R}^{d}, d=1,2$, is convex, there exists $\bar{D}_{0} \subset D$ and $\ell \in \mathbb{N}$ such that

$$
\int_{D} e^{u} d x \leq(\ell+1) \int_{D_{0}} e^{u} d x
$$

for any $0 \leq t<T_{\max }$ and for some positive integer $m$, where $T_{\max } \leq \infty$ is the maximal existence time of $u$.

Proof. For any $y \in \partial D$ we define the hyperplane

$$
\mathscr{T}(\mu, y):=\left\{x \in \mathbb{R}^{d}:(x, \nu(y))_{d}=\mu\right\},
$$

where $(\cdot, \cdot)_{d}$ stands for the inner product in $\mathbb{R}^{d}$.

We can find $\mu_{0}$ such that $\mathscr{T}\left(\mu_{0}, y\right)$ coincides with the tangent hyperplane to $D$ at $y$ and $y \in \mathscr{T}\left(\mu_{0}, y\right) \cap \bar{D}$ (when $D$ is strictly convex then $\mathscr{T}\left(\mu_{0}, y\right) \cap \bar{D}=\{y\}$ ). 
Since $D$ is a bounded set there exists $\mu_{1}<\mu_{0}$ such that $\mathscr{T}(\mu, y) \cap \bar{D}=\emptyset$ for $\mu>\mu_{0}$ and $\mu<\mu_{0}-\mu_{1}$.

We define

$$
\Sigma(\mu, y):=\left\{x \in D: \mu<(x, \nu(y))_{d}<\mu_{0}\right\}
$$

while by $\Sigma^{\prime}(\mu, y)$ we denote the reflection of $\Sigma(\mu, y)$ across $\mathscr{T}(\mu, y)$. Now using the convexity of $D$ we can choose $\bar{\mu}$ sufficiently close to $\mu_{0}$ so that $\Sigma^{\prime}(\bar{\mu}, y) \subset D$.

Applying Theorem 3.4 we derive for any $y \in \partial D$

$$
\frac{\partial u(y, t)}{\partial \nu}=(\nabla u(y, t), \nu(y))_{d}<0, \quad \text { for any } \quad t \geq t_{0}>0 .
$$

By the spatial regularity of $u$, see (1.8), we can find a neighbourhood of $y$, say $\mathscr{N}_{y}$, such that

$$
\frac{\partial u\left(x, t_{0}\right)}{\partial \nu}=\left(\nabla u\left(x, t_{0}\right), \nu(y)\right)_{d}<0, \quad \text { for any } \quad x \in \mathscr{N}_{y} .
$$

We consider now a coordinate system centered at $y$ and defined by $\left(y ; \nu(y), \mathscr{T}\left(\mu_{0}, y\right)\right)$ such that every $x \in \mathbb{R}^{d}$ is expressed as $x=\left(x_{\nu}, x_{\mathscr{T}}\right)$, where $x_{\nu}$ is the component in the direction of $\nu(y)$ while $x_{\mathscr{T}}$ stands for the component in the direction of the hyperplane $\mathscr{T}\left(\mu_{0}, y\right)$.

Let us define the cylinder $C_{\delta}(y)=\left\{y \in \mathbb{R}^{d}:\left|x_{\nu}\right|<\delta,\left|x_{\mathscr{T}}\right|<\delta\right\}$. We may pick $\delta>0$ small enough so that the reflection of $\overline{C_{\delta}(y)} \cap D$ across $\mathscr{T}(\bar{\mu}, y)$, denoted by $C_{\delta}^{\prime}(y)$, is compact in $D$.

Set $K_{y}=\mathscr{T}\left(\mu_{0}, y\right) \cap \bar{D}$, then $K_{y}$ is a compact convex set and $K_{y}=\bigcap_{\mu<\mu_{0}} \Sigma(\mu, y)$. Every $\hat{y} \in K_{y}$ has the same exterior normal $\nu(y)$. Then we can define an open neighbourhood of $\hat{y}$ of the shape $C_{\delta}(\hat{y})$ and on which $\left(\nabla u\left(\hat{y}, t_{0}\right), \nu(y)\right)_{d}<0$. Moreover, $K_{y} \subset \bigcup_{\hat{y}} C_{\delta}(\hat{y})$ and since $K_{y}$ is compact we can extract a finite cover of $C_{\delta}(\hat{y})$, say $B=\bigcup_{i=1}^{n} C_{\delta}\left(\hat{y}_{i}\right)$ which contains $K_{y}$, for some positive integer $n=n(y)$.

Since $D$ is convex we can find $\mu<\mu_{0}$ such that $\Sigma(\mu, y) \subset B$ and $\Sigma^{\prime}\left(\rho_{0}, y\right) \subset$ $D, \Sigma\left(\rho_{0}, y\right) \cup \Sigma^{\prime}\left(\rho_{0}, y\right) \subset B$ for $\rho_{0}=\frac{\mu+\mu_{0}}{2}$. (Note that if $D$ is strictly convex then the above construction is unnecessary).

We now set $z(x, t)=z\left(x_{\nu}, x_{\mathscr{T}}, t\right)=u\left(2 \rho_{0}-x_{\nu}, x_{\mathscr{T}}, t\right)$ for $x \in \Sigma\left(\rho_{0}, y\right)$; actually $z$ is the reflection of $u$ across $T\left(\rho_{0}, y\right)$. Then $z$ satisfies

$$
\begin{aligned}
& d z(\cdot, t)=\left[A z(\cdot, t)+K(t) e^{z(\cdot, t)}\right] d t+\sigma(z(\cdot, t)) d W(t), \quad \text { on } \quad \Sigma\left(\rho_{0}, y\right) \times\left(0, T_{\text {max }}\right) \\
& z \geq u \geq 0 \quad \text { on } \partial \Gamma_{1}=\left(\partial D \cap \Sigma\left(\rho_{0}, y\right)\right) \times\left(0, T_{\max }\right), \\
& z_{t}=u_{t} \quad \text { on } \partial \Gamma_{2}=\left(D \cap \mathscr{T}\left(\rho_{0}, y\right)\right) \times\left(0, T_{\max }\right) .
\end{aligned}
$$

Thus $z$ and $u$ satisfy the same SPDE on $\Sigma\left(\rho_{0}, y\right) \times\left(0, T_{\max }\right)$ while $z \geq u$ on $\partial \Gamma_{1} \cup \partial \Gamma_{2}$ and $z\left(\cdot, t_{0}\right) \geq u\left(\cdot, t_{0}\right)$ on $\Sigma\left(\rho_{0}, y\right)$, hence by the comparison principle, see $[1,11]$, we deduce that $z \geq u$ almost surely (a.s.) on $\Sigma\left(\rho_{0}, y\right) \times\left(0, T_{\max }\right)$.

Note that $\Sigma\left(\rho_{0}, y\right)$ contains an open set of the type $C_{\delta}(y) \cap D$ and if we choose $\delta<$ $\mu_{0}-\rho_{0}$ then the reflection of $C_{\delta}(y) \cap D$ across $\mathscr{T}\left(\rho_{0}, y\right)$ has a compact closure in $D$. We can repeat the above construction for any $y \in \partial D$ and the collection of all cylinders 
$\left\{C_{\delta}(y)\right\}_{y \in \partial D}$ makes up an open cover of $\partial D$, and we can extract a finite subcover denoted by $C_{\delta}\left(y_{1}\right), \ldots, C_{\delta}\left(y_{\ell}\right)$ such that $\partial D \subseteq C_{\delta}\left(y_{1}\right) \cup \ldots \cup C_{\delta}\left(y_{\ell}\right)$.

$$
\text { Set } \begin{aligned}
D_{0}=D \backslash \bigcup_{i=1}^{\ell} & C_{\delta}\left(y_{i}\right) \text {, then } \bar{D}_{0} \subset D \text { and we derive } \\
\int_{D} u d x & \leq \int_{D_{0}} u d x+\sum_{i=1}^{\ell} \int_{C_{\delta}\left(y_{i}\right) \cap D} u d x \leq \int_{D_{0}} u d x+\sum_{i=1}^{\ell} \int_{C_{\delta}\left(y_{i}\right) \cap D} z d x \\
& \leq \int_{D_{0}} u d x+\sum_{i=1}^{\ell} \int_{C_{\delta}^{\prime}\left(y_{i}\right)} z d x=\int_{D_{0}} u d x+\sum_{i=1}^{\ell} \int_{C_{\delta}^{\prime}\left(y_{i}\right)} u d x \\
& \leq \int_{D_{0}} u d x+\ell \int_{D_{0}} u d x \leq(\ell+1) \int_{D_{0}} u d x,
\end{aligned}
$$

taking also into account that $u \leq z$ on $C_{\delta}\left(y_{i}\right) \cap D$ and $u=z$ on $C_{\delta}^{\prime}\left(y_{i}\right)$ by reflection.

Now since $e^{s}$ is increasing we deduce

$$
\int_{D} e^{u} d x \leq(\ell+1) \int_{D_{0}} e^{u} d x
$$

and the proof of Lemma is now complete.

\section{EXPLOSION IN FINITE TIME}

In this section we prove that the local-in-time solution of (1.5)-(1.6) cannot be extended as a global-in-time solution under some circumstances and hence finite-time explosion occurs. To this end we employ a widely utilized classical technique of Kaplan ([16]), which essentially relies on testing (1.7) by positive Dirichlet eigenfunctions of the Laplacian.

Our first blow-up result is valid when the parameter $\lambda$ is large enough.

Theorem 4.1. Suppose that (1.5)-(1.6) has a unique local-in-time solution $u$. Then $u$ explodes in finite time for sufficiently large values of the parameter $\lambda$, provided that $\xi \in$ $L^{2}\left(\Omega ; L^{2}(D)\right)$.

Proof. We follow the proof for the deterministic case in [17].

Let $\lambda_{1}, \phi_{1}(x)$ be the first eigenvalue and the corresponding eigenfunction respectively of the operator $-\Delta$ with $\mathscr{D}(A)=L^{2}(D)$, i.e. $\phi$ satisfies

$$
\begin{aligned}
& \Delta \phi_{1}=-\lambda_{1} \phi_{1}, x \in D \\
& \phi_{1}=0, x \in \partial D .
\end{aligned}
$$

Since it is well known that $\phi_{1}$ has a constant sign on $D$ we can take $\phi_{1} \geq 0$ on $\bar{D}$ and normalized such that

$$
\int_{D} \phi_{1} d x=1
$$


Recall that the solution $u_{t}$, due to (1.7), can be expressed as

$$
u(\cdot, t)=\xi+\int_{0}^{t} A u(\cdot, s) d s+\int_{0}^{t} \frac{e^{u(\cdot, s)}}{\left(\int_{D} e^{u(x, s)} d x\right)^{p}} d s+\int_{0}^{t} \sigma(u(\cdot, s)) d W(s),
$$

then by multiplying (4.2) by $\phi_{1}(x)$ and integrating over $D$ we derive

$$
\begin{aligned}
\hat{u}(t): \quad & =\int_{D} u \phi_{1} d x \\
& =\int_{D} \xi \phi_{1} d x+\int_{0}^{t} \int_{D} A u \phi_{1} d x d s \\
& +\lambda \int_{0}^{t} \int_{D} \frac{e^{u} \phi_{1}}{\left(\int_{D} e^{u} d x\right)^{p}} d x d s+\int_{0}^{t} \int_{D} \sigma(u) \phi_{1} d x d W(s) .
\end{aligned}
$$

Now taking the expectation over (4.3) we derive

$$
\begin{aligned}
\mathbb{E}[\hat{u}(t)] & =\mathbb{E}\left[\int_{D} \xi \phi_{1} d x\right]+\mathbb{E}\left[\int_{0}^{t} \int_{D} A u \phi_{1} d x d s\right] \\
& +\lambda \mathbb{E}\left[\int_{0}^{t} \int_{D} \frac{e^{u} \phi_{1}}{\left(\int_{D} e^{u} d x\right)^{p}} d x d s\right] \\
& +\mathbb{E}\left[\int_{0}^{t} \int_{D} \sigma(u) \phi_{1} d x d W(s)\right] .
\end{aligned}
$$

Note that

$$
\begin{aligned}
\int_{D} A u \phi_{1} d x & =\left\langle A u, \phi_{1}\right\rangle=\left\langle u, A \phi_{1}\right\rangle=\left\langle u,-\lambda_{1} \phi_{1}\right\rangle \\
& =-\lambda_{1} \int_{D} u \phi_{1} d x,
\end{aligned}
$$

since $A$ is a self-adjoint operator and

$$
\mathbb{E}\left[\int_{0}^{t} \int_{D} \sigma(u) \phi_{1}(x) d x d W(s)\right]=0
$$

due to the fact that $W(t)$ is a Wiener process.

Set $\Psi(t)=\mathbb{E}[\hat{u}(t)]$, then by interchanging the order of expectation and integration, by virtue of Fubini's theorerm, we deduce for $t \geq 0$,

$$
\Psi(t)=\Psi_{0}-\lambda_{1} \int_{0}^{t} \Psi(s) d s+\lambda \mathbb{E}\left[\int_{0}^{t} \int_{D} \frac{e^{u} \phi_{1}}{\left(\int_{D} e^{u} d x\right)^{p}} d x d s\right],
$$

where $\Psi_{0}=\mathbb{E}\left(\left\langle\xi, \phi_{1}\right\rangle\right)$, or equivalently the differential form,

$$
\frac{d \Psi}{d t}=-\lambda_{1} \Psi(t)+\lambda \mathbb{E}\left[K(t) \int_{D} e^{u} \phi_{1} d x\right], \quad t>0, \quad \Psi(0)=\Psi_{0},
$$

recalling that $K(t)=\left(\int_{D} e^{u} d x\right)^{-p}$. 
By Lemma 3.5, we can construct $D_{0} \subset D$ with $\bar{D}_{0} \subset D$ such that

$$
\int_{D} e^{u} d x \leq(\ell+1) \int_{D_{0}} e^{u} d x
$$

for some $\ell \in \mathbb{N}$. Let $m=\inf _{x \in D_{0}} \phi_{1}(x)$, then by using the fact that $\bar{D}_{0} \subset D$ and the maximum principle, we have $m>0$. Hence

$$
\int_{D} e^{u} d x \leq \frac{\ell+1}{m} \int_{D_{0}} e^{u} \phi_{1} d x \leq \frac{\ell+1}{m} \int_{D} e^{u} \phi_{1} d x
$$

and so

$$
K(t)=\left(\int_{D} e^{u} d x\right)^{-p} \geq C\left(\int_{D} e^{u} \phi_{1} d x\right)^{-p}
$$

where

$$
C=\left(\frac{m}{\ell+1}\right)^{p}
$$

Therefore by virtue of (4.7) and applying Jensen's inequality twice, since both $e^{s}$ and $e^{(1-p) s}$ are convex functions for $0<p<1$, we deduce

$$
\begin{aligned}
\mathbb{E}\left[K(t) \int_{D} e^{u} \phi_{1} d x\right] & \geq \mathbb{E}\left[C\left(\int_{D} e^{u} \phi_{1} d x\right)^{1-p}\right] \\
& \geq C e^{(1-p) \mathbb{E}[\hat{u}(t)]} \\
& \geq C e^{(1-p) \mathbb{E}[\hat{u}(t)]} \\
& =C e^{(1-p) \Psi(t)} .
\end{aligned}
$$

Combining (4.6) with (4.9),

$$
\frac{d \Psi(t)}{d t} \geq-\lambda_{1} \Psi(t)+\lambda C e^{(1-p) \Psi(t)}, \quad t>0, \quad \Psi(0)=\Psi_{0}
$$

and by choosing $\lambda>\lambda_{1} B / C$, where $0<B=\sup _{s>\Psi(0)} s e^{(p-1) s}<\infty$, we have

$$
\begin{aligned}
t & \leq \int_{\Psi(0)}^{\Psi(t)} \frac{d s}{\lambda C e^{(1-p) s}-\lambda_{1} s} \leq \frac{1}{\Lambda} \int_{\Psi(0)}^{\Psi(t)} \frac{d s}{e^{(1-p) s}} \\
& <\frac{1}{\Lambda} \int_{\Psi(0)}^{\infty} \frac{d s}{e^{(1-p) s}}<\infty
\end{aligned}
$$

for

$$
0<\Lambda \leq \lambda C-\lambda_{1} B<\infty
$$

Thus $\Psi(t)$ explodes in finite time, i.e. $\Psi(t) \rightarrow \infty$ as $t \rightarrow T^{*}$ where $T^{*}$ is estimated as

$$
T^{*} \leq \int_{\Psi(0)}^{\infty} \frac{d s}{\lambda C e^{(1-p) s}-\lambda_{1} s} \leq \frac{1}{\Lambda} \int_{\Psi(0)}^{\infty} \frac{d s}{e^{(1-p) s}}<\infty
$$


Indeed, the finite explosion of $\Psi(t)$ guarantees the finite explosion of $u(x, t)$ as well since

$$
\Psi(t)=\mathbb{E}\left[\int_{D} u \phi_{1} d x\right] \leq \mathbb{E}\left[\|u(\cdot, t)\|_{\infty}\right]
$$

implies that $\mathbb{E}\left[\|u(\cdot, t)\|_{\infty}\right] \rightarrow \infty$ as $t \rightarrow T_{e} \leq T^{*}$.

The proof of the Theorem is complete.

Finite time explosion occurs for large enough initial data as well. Indeed the following result is valid.

Theorem 4.2. Suppose that (1.5)-(1.6) has a unique local-in-time solution u. Assume also that initial data $\xi \in L^{2}\left(\Omega ; L^{2}(D)\right)$ satisfy

$$
\Psi(0)=\Psi_{0}:=\mathbb{E}\left(\int_{D} \xi \phi_{1} d x\right)>\zeta,
$$

where $\zeta=\zeta(\lambda)$ is the largest root of the equation

$$
g(s):=\lambda C e^{(1-p) s}-\lambda_{1} s=0,
$$

and $C$ is the constant given by (4.8). Then the solution $u$ of (1.5) - (1.6) explodes in finite time.

Proof. Following the same steps as in the proof of Theorem 4.1 we obtain that $\Psi(t)=$ $\mathbb{E}\left[\int_{D} u \phi_{1} d x\right]$ satisfies the differential inequality

$$
\frac{d \Psi(t)}{d t} \geq-\lambda_{1} \Psi(t)+\lambda C e^{(1-p) \Psi(t)}=g(\Psi(t)), \quad t>0, \quad \Psi(0)=\Psi_{0}
$$

where constant $C$ is defined by (4.8).

Let $\zeta=\zeta(\lambda)>0$ be the largest root of the equation $g(s)$ then $g(s)>0$ for any $s>\zeta$; otherwise if $\zeta=0$ then $g(s)>0$ for any $s>0$.

Then by choosing $\Psi_{0}>\zeta$ we deduce

$$
\int_{\Psi_{0}}^{\infty} \frac{d s}{g(s)} \leq \frac{1}{\Lambda_{1}} \int_{\Psi_{0}}^{\infty} \frac{d s}{e^{(1-p) s}}<\infty,
$$

for some positive constant $\Lambda_{1}$. But the above relation guarantees that $\Psi(t)$ explodes in finite time $T^{*}<\infty$, where $T^{*}$ estimated as in (4.12). The latter actually implies that $u$ also explodes in finite time $T_{e} \leq T^{*}$.

Remark 4.3. Theorems 4.1 and 4.2 both imply explosion of the mean $L^{q}$-norm for any $q \geq 1$ as well. Indeed, since $\phi_{1}$ is bounded and continuous on $\bar{D}$ by applying Hölder's inequality for each $q \geq 1$ we derive

$$
\Psi(t) \leq C_{q} \mathbb{E}\left[\left(\int_{D}|u|^{q} d x\right)^{1 / q}\right]
$$


for $C_{q}=\left(\int_{D}\left|\phi_{1}\right|^{r} d x\right)^{1 / r}$ with $r=q /(q-1)$, which actually yields that the mean $L^{q}-$ norm explodes in finite time $T_{q} \leq T_{e}$.

\section{REFERENCES}

[1] S. Assing Comparison of systems of stochastic partial differential equations, Stochastic Processes and their Applications 82 (1999), 259-282.

[2] J. Bebernes Non-local models of shear banding, R. Spigler (ed.), Applied and Industrial Mathematics, Venice-2, 1998, 33-46, Dordrecht (2000) Kluwer Academic Publishers.

[3] T.J. BuRns, A mechanism for shear band formation in the high strain-rate torsion test, Journal of Applied Mechanics 57 (1990), 836-844.

[4] T.J. Burns, Connections between localized behavior in plasticity and in combustion, in:R.C. Batra, H.M. Zbib (Eds.), Material Instabilities Theory and Application, The American Soc. of Mech. Engineers, New York, Book No. G00895, (1994), 87-93.

[5] T.J. BuRns, Does a shear band result from a thermal explosion?, Mechanics of Material, 17 (1994), 261-271.

[6] P-L CHow, Stochastic Partial Differential Equations, Chapman and Hall/CRC, Boca Raton, FL, ; London, 2007.

[7] P-L CHOw, Explosive solutions of stochastic reaction-diffusion equations in mean $L^{p}$-norm, Journal of Differetial Equations, 250 (2011), 2567-2580.

[8] P-L Chow, Unbounded positive solutions of nonlinear parabolic Itô equations, Communications on Stochastic Analysis, 3 (2009), 211-222.

[9] A. Debussche, S. De Moor And M. Hofmanová, A regularity result for quasilinear stochastic partial differential equations of parabolic type, SIAM Journal on Mathematical Analysis, to appear.

[10] L. Denis, A. Matoussi And L. Stoica, Maximum principle for quasi-linear SPDE's on a bounded domain without regularity assumptions, Stochastic Processes and their Applications, 123 (2013), $1104-1137$.

[11] L. Denis, A. Matoussi And L. Stoica, Maximum principle and comparison theorem for quasilinear stochastic PDE's, Electronic Journal of Probability 14 (2009), 500-530.

[12] C. Donati-Martin \& N. Privault, White noise driven SPDEs with reflection, Probability Theory and Related Fields 95 (1993), 1-24.

[13] M. Dozzi \& J.A. LòPez-Mimbela, Finite-time blowup and existence of global positive solutions of a semi-linear SPDE, Stochastic Processes and their Applications 120 (2010), 767-776.

[14] A. Friedman, Partial Differential Equations of Parabolic Type, 1983, New York, Prentice-Hall Inc.

[15] N. Ikeda And S. Watanabe, Stochastic Differential Equations and Diffusion Processes, 2nd Edition 1989, North-Holland/Kodansha, Amsterdam/Tokyo.

[16] S. KAPLAN, On the growth of solutions of quasilinear parabolic equations, Communcications of Pure and Applied Mathematics 16 (1963), 327-330.

[17] N. I. Kavallaris And D. E. Tzanetis, On the blow-up of a non-local parabolic problem, Applied Mathematics Letters 19 (2006), 921-925.

[18] N. I. KaVallaris AND Y. Yan, On the finite-time bloww-up of a non-local stochastic parabolic problem, preprint.

[19] A.A. Lacey and D.E. Tzanetis, Global unbounded solutions to a parabolic equation, Journal of Differetial Equations, 101 (1993), 80-102.

[20] A. Marchand And J. Duffy, An experimental study of the formation of adiabatic shear bands in a structural steel, Journal of the Mechanics and Physics of Solids, 36 (1988), 251-283.

[21] J. Smoller, Shock Waves and Reaction-Diffusion Equations, 2nd Edition 1983, New York, SpringerVerlag. 
[22] T. SHIGA, Two contrasting properties of solutions for one-dimesnoonal stochastic stochstic partial differential equations, Canadian Journal of Mathematics 46 (1994), 415-437.

Department of Mathematics, University of Chester, Thornton Science Park Pool Lane, InCE, Chester CH2 4NU, UK

E-mail address: n.kavallaris@chester.ac.uk 